\title{
Phase Behavior of Model Lipid Bilayers ${ }^{\dagger}$
}

\author{
Marieke Kranenburg ${ }^{\ddagger}$ and Berend Smit $*, \ldots, \$$ \\ The Van 't Hoff Institute for Molecular Sciences, University of Amsterdam, Nieuwe Achtergracht 166, \\ 1018 WV Amsterdam, The Netherlands, and CECAM (Centre Européen de Calcul Atomique Moléculaire), \\ Ecole Normale Supérieure, 46 Allée d'Italie, 69364 Lyon Cedex 7, France
}

Received: September 17, 2004; In Final Form: November 21, 2004

\begin{abstract}
We investigated the phase behavior of double-tail lipids, as a function of temperature, headgroup interaction and tail length. At low values of the head-head repulsion parameter $a_{\mathrm{hh}}$, the bilayer undergoes with increasing temperature the transitions from the subgel phase $L_{\mathrm{c}}$ via the flat gel phase $L_{\beta}$ to the fluid phase $L_{\alpha}$. For higher values of $a_{\mathrm{hh}}$, the transition from the $L_{\mathrm{c}}$ to the $L_{\alpha}$ phase occurs via the tilted gel phase $L_{\beta^{\prime}}$ and the rippled phase $P_{\beta^{\prime}}$. The occurrence of the $L_{\beta^{\prime}}$ phase depends on tail length. We find that the rippled structure $\left(P_{\beta^{\prime}}\right)$ occurs if the headgroups are sufficiently surrounded by water and that the ripple is a coexistence between the $L_{\mathrm{c}}$ or $L_{\beta^{\prime}}$ phase and the $L_{\alpha}$ phase. The anomalous swelling, observed at the $P_{\beta^{\prime}} \rightarrow L_{\alpha}$ transition, is not directly related to the rippled phase, but a consequence of conformational changes of the tails.
\end{abstract}

\section{Introduction}

Knowledge on the structure of lipid bilayers is important for our understanding of the functioning of biological membranes. Phospholipids are the main components of biological membranes. They can self-assemble in different structures, of which the lipid bilayer is the most important structure in nature. The phospholipid bilayer is surrounding the cell and protects the cell from it's environment, ensuring that different processes in the cell can occur. In a bilayer, the hydrophilic headgroup of a phospholipid is oriented toward the water phase, and the hydrocarbon tails form the inner hydrophobic part, in which the terminal methyl groups from the opposing layers face each other. Much research, both experimentally and theoretically, is devoted to the phase diagram of the phospholipid bilayer (for a review, see refs 1 and 2).

A phospholipid bilayer knows many different phases, depending on temperature, pressure, and hydration, and on its structural properties, such as the length of the hydrocarbon tails and the composition of the headgroup (see Figure 1). For the most common phospholipids, the low-temperature phase is the subgel $L_{\mathrm{c}}$, in which the hydrocarbon tails are highly ordered and show a tilt with respect to the bilayer. ${ }^{1}$ Upon heating the subgel transforms to a lamellar gel phase. Dependent on the structural composition of the lipid headgroup, the gel phase is the $L_{\beta}$ phase (for example, for phosphatidylethanolamines or PEs) or the $L_{\beta^{\prime}}$ phase (for example, for phosphatidylcholines or $\mathrm{PCs})$. In these gel phases, the bilayer is more hydrated than in the $L_{\mathrm{c}}$ phase and the hydrocarbon tails still show a high order, but less than in the $L_{\mathrm{c}}$ phase. In the $L_{\beta}$ phase the tails are ordered parallel to the bilayer normal, while in the $\mathrm{L}_{\beta^{\prime}}$ phase the tails show a tilt angle with respect to the bilayer normal. At higher temperature, the gel phase undergoes a transition to the $L_{\alpha}$ phase, which is also called the liquid crystalline or fluid phase; the tails are disordered and do not show any tilt. This $L_{\alpha}$ phase is physiologically the most important phase.

\footnotetext{
† Part of the special issue "David Chandler Festschrift"

The Van 't Hoff Institute for Molecular Sciences, University of Amsterdam.

$\S$ CECAM/Ecole Normale Supérieure de Lyon.
}
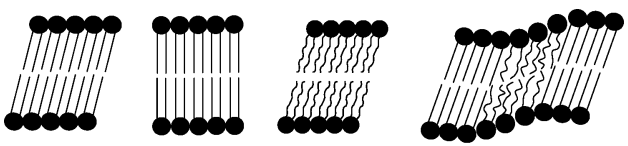
(a) $L_{c}$
(b) $L_{\beta}$
(c) $L_{\beta^{\prime}}$

(d) $P_{\beta^{\prime}}$

(e) $L_{\alpha}$

Figure 1. Schematical drawings of the various bilayer phases. The characteristics of these phases are explained in the text. The filled circles represent the hydrophilic headgroup of a phospholipid and the lines represent the hydrophobic tails.

For some lipids, the transition from the ordered gel phase to the disordered liquid crystalline phase occurs in two steps. First, a transition from the gel phase to the rippled phase $P_{\beta^{\prime}}$ takes place (i.e., the pretransition). This transition is followed by the melting of the bilayer from the $P_{\beta^{\prime}}$ to the $L_{\alpha}$ phase, which is called the main transition. The rippled phase is characterized by a long-wavelength rippling of the bilayer and an (anomalous) swelling of the membrane. The hydrophobic chains are ordered and there is a preferred tilt angle with respect to the bilayer normal. The temperature interval between the pretransition and the main transition decreases with increasing chain length. For chains containing more than 20 carbon atoms the pretransition is not observed. It is assumed that this transition disappears completely or that the temperature interval between the pretransition and the main transition is too small to be observed. ${ }^{3}$ The rippled phase is only observed in bilayers containing PCs, of which the low-temperature phase is the $L_{\beta^{\prime}}$. Phosphatidylethanolamines (PEs) and glucolipids, of which the lowtemperature phase is the untilted $L_{\beta}$, do not display a pretransition (ref 4 and references therein).

This rippled phase has attracted the attention of many groups, from the moment it was first observed by Tardieu et al. in 1973. ${ }^{5}$ After this first observation, many studies, both theoretically and experimentally, have been addressed to the question how such a corrugated $P_{\beta^{\prime}}$ phase can exist, while the low-temperature gel phase $L_{\beta^{\prime}}$ and the high-temperature fluid phase $L_{\alpha}$ are flat. The general model depicts the ripple as a asymmetric sawtooth, ${ }^{5-9}$ but in some models it is also proposed that the ripple is a sinusoïdal. ${ }^{4,10-13}$ The logical next question is then how the 
sawtooth or the sinusoïdal can occur. Explanations can be found in variations of the thickness of the bilayer due to changes in tilt angles of the hydrophobic chains, ${ }^{12-18}$ and coexistence between the fluid $L_{\alpha}$ phase and the gel phase $L_{\beta^{\prime}} \cdot{ }^{4,7,19-23}$ Also, it is not very clear if the (anomalous) swelling of a membrane is coupled to the formation of the rippled phase $e^{2,24,25}$ and if the rippled phase only exists in multilayers or if it is also present in a single bilayer system. ${ }^{26-28}$

Despite all investigations, an explanation of the formation of the rippled phase at a molecular level is still lacking. We use computer simulations to study the phase behavior of phospholipid bilayers. Simulations allow us to investigate the bilayer at a molecular level, which is not always possible experimentally. We use a combined technique of Dissipative Particle Dynamics (DPD) and Monte Carlo simulations, in which we impose the bilayer to adopt a tensionless configuration. This method allows us to observe directly phase transitions in which the area per lipid changes.

The phase behavior of membranes depends on the structural properties of the phospholipid. Therefore, we study the phase behavior as a function of tail length and headgroup interaction. Here, we apply a mesoscopic model consisting of three hydrophilic beads and two hydrophobic tails. A short communication on these results has been published previouly. ${ }^{29}$ The resulting phase diagrams are presented in section 3 . We will show that we can reproduce the various phases of the phospholipid bilayer, dependent on temperature and head-head interaction. While for the shortest lipid studied the $L_{\beta^{\prime}}$ phase is not present, this phase appears for longer tails and its stability increases with increasing tail length. We show that the anomalous swelling is not directly related to the formation of the rippled phase and finally, we discuss the structure of the ripple. We conclude that the key factor in the formation of the rippled phase is a frustration of the surface area of the headgroup with the packing of the hydrophobic tails.

\section{Model and Computational Details}

In our mesoscopic lipid-water model, we distinguish three types of particles, $w, h$, and $t$, to mimic water and the headand tail-atoms of a lipid, respectively. The hydrophilic and hydrophobic particles interact via a soft-repulsion model commonly used in dissipative particle dynamics (DPD). ${ }^{30,31}$ In a DPD simulation, the atoms are lumped together such that a DPD particle represents the center of mass of a cluster of atoms. The total force on such a particle consists of dissipative, random, and conservative forces. The dissipative random forces are chosen such that a proper canonical distribution is sampled. ${ }^{31}$ For the conservative force, we use the conventional softrepulsive forces, given by

$$
F_{\mathrm{ij}}^{\mathrm{C}}= \begin{cases}a_{i j}\left(1-r_{i j} / r_{c}\right) & r_{i j}<r_{\mathrm{c}} \\ 0 & r_{i j} \geq r_{\mathrm{c}}\end{cases}
$$

where $r_{i j}$ is the distance between particles $i$ and $j, a_{i j}$ is the parameter characterizing the interaction between two particles, and $r_{\mathrm{c}}$ is the cutoff radius. The DPD parameters are related the compressibility of water and to Flory-Huggins solubility parameters such that a reasonable description of the thermodynamics of the real system can be obtained. ${ }^{30}$

We consider lipids with three head segments and two tails with variable length (see Figure 2). We assume that a DPD particle occupies a volume of $90 \AA^{3}$, which results in a mapping in which a tail length of five beads corresponds to the phospholipid DMPC. For a correct description of the experi-

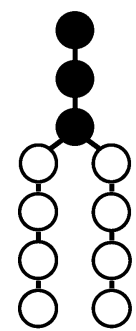

$\mathrm{h}_{3}\left(\mathrm{t}_{4}\right)_{2}$

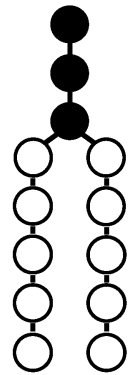

$\mathrm{h}_{3}\left(\mathrm{t}_{5}\right)_{2}$

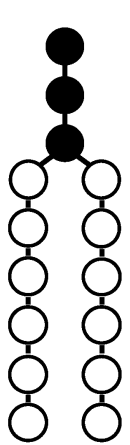

$\mathrm{h}_{3}\left(\mathrm{t}_{6}\right)_{2}$

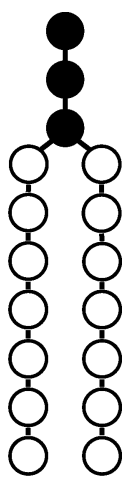

$\mathrm{h}_{3}\left(\mathrm{t}_{7}\right)_{2}$
Figure 2. Models of the lipids used in this study with their nomenclature; the black particles represent the head beads and the white particles the tail beads.

mental chain length dependence of the area per lipid, it is essential to properly reproduce the conformations of the lipid. Molecular Dynamics simulations of a single phospholipid in water using a realistic all-atom representation are used to generate configurations of the lipid, which were subsequently used to optimize the intramolecular interactions (bond-bending and bond-vibration) of the DPD model. A detailed description of this procedure can be found in ref 32. In refs 33 and 34 this model has been extended to include alcohol. We used similar parameters as Groot $^{30}$ for the soft-repulsions in the conservative DPD interactions $\left(a_{\mathrm{ww}}=a_{\mathrm{tt}}=25, a_{\mathrm{ht}}=a_{\mathrm{wt}}=80\right.$, and $a_{\mathrm{hw}}=$ 15). In addition, we vary the head-head interactions, $a_{\mathrm{hh}}$ to study the effect of changing the interactions between the headgroups of a lipid. Experimentally, the head-head interactions can be changed by, for example, modifying the chemical nature of the headgroup or adding salt to the system. The intramolecular interactions include a bond-bending potential

$$
U_{\theta}=(1 / 2) k_{\theta}\left(\theta-\theta_{0}\right)^{2}
$$

with $k_{\theta}=6$ and $\theta_{0}=180^{\circ}$ for the tails and between the tails and the headgroup $k_{\theta}=6$ and $\theta_{0}=90^{\circ}$. Two consecutive beads are connected by harmonic springs

$$
U_{\mathrm{r}}=(1 / 2) k_{\mathrm{r}}\left(r-r_{0}\right)^{2}
$$

with spring constant $k_{\mathrm{r}}=100$ and equilibrium distance $r_{0}=$ 0.7 .

A biological membrane is not subject to external constraints and therefore adopts a configuration which is tensionless. Lipowski and co-workers ${ }^{35,36}$ emphasize the importance of simulating at exactly the area for which the interfacial tension is zero and determine this area iteratively. We use a different approach in which we mimic the experiment by simulating an ensemble in which we impose the interfacial tension. After a randomly selected number of DPD steps we perform a Monte Carlo move in which we change the area of our bilayer in such a way that the total volume of the system remains constant. This move is accepted with a probability ${ }^{37}$

$$
\operatorname{acc}(\mathrm{o} \rightarrow \mathrm{n})=\min \left(1, \frac{\exp \left\{-\beta\left[U(\mathrm{n})-\gamma A_{\mathrm{n}}\right]\right\}}{\exp \left\{-\beta\left[U(\mathrm{o})-\gamma A_{\mathrm{o}}\right]\right\}}\right)
$$

where $U(o)$ and $U(n)$ indicate the energy of the old and the new configuration, respectively, $\gamma$ the interfacial tension, $A$ the area of the bilayer and $\beta=1 / k_{\mathrm{B}} T$. To obtain the tensionless state of the bilayer is set to zero to ensure that the membrane 
adopts the equilibrium area per lipid. ${ }^{37-39}$ The importance of this method is that it allows us to observe directly phase transitions in which the area per lipid changes.

Simulations were performed on a bilayer containing 800 lipids. 8000-15000 water particles are added to ensure that a bilayer does not have any interaction with its periodic image in the horizontal direction. After the formation of the bilayer, by applying DPD steps only, we allowed the bilayer to adopt a tensionless configuration by applying both DPD and Monte Carlo, in which the area of the bilayer is changed. The overall density of the system is $\rho=3$. A typical simulation required 100000 cycles of which 20000 cycles were needed for equilibration. Per cycle it is chosen with a probability of $70 \%$ whether to perform 50 DPD time steps or to make an attempt to change the area of the box. To test the reproducibility of our simulations, we repeated the self-assembly from different initial condition and by heating of the system and subsequent cooling. These simulations with different initial conditions gave within the statistical uncertainly identical results. More details on the simulations can be found in refs 37 and 39 .

To characterize the different phases we used the area per lipid, the order of the tails, the tilt angle, and the thickness of the hydrophobic part of the bilayer as order parameters. The area per lipid $A_{1}$ is simply computed by dividing the total area in the $y z$-plane at $\gamma=0$ by half the number of lipids in the bilayer. On average the number of lipids in each side of the bilayer is equal.

The order of the tails $S_{\text {tail }}$ is defined as

$$
S_{\text {tail }}=\frac{1}{2}\left\langle 3 \cos ^{2} \theta-1\right\rangle
$$

where $\theta$ is the angle between vector connecting the first and the last bead in the tail and the bilayer normal (the tilt angle). The order parameter has a value 1 if the vector is parallel to the bilayer normal, a value 0 if the orientation is random, and -0.5 if the bond is on average perpendicular. Finally, we compute the thickness of the hydrophobic core and the bilayer thickness as the average distance between the headgroups of two opposing lipids. In case of the hydrophobic thickness, $D_{\mathrm{c}}$, the bead connecting the two tails is used as a reference bead, whereas the bilayer thickness, $D_{\mathrm{b}}$, is computed using the last bead of the headgroup as the reference bead.

By studying the behavior of these quantities as function of temperature and head-head repulsion parameter we determine the phase boundaries from the inflection points of these curves. The temperature at which the chains get disordered is the same as the temperature of the inflection point in $A_{1}$ and $D_{\mathrm{c}}$. Although the overall systems we are simulating are quite large, the phase transitions are quasi two-dimensional and from a "twodimensional viewpoint of view" our systems are much smaller. As a consequence, the transitions we observe are very gradual and much larger systems will be required to carefully analyze the order of the transition.

In our simulations we use the conventional reduced units, i.e., using $r_{\mathrm{c}}$ as the unit of length and $a=1$ as the unit of energy. For some properties it is interesting to make a direct comparison with experiments. The conversion factor for the length scale from the assumption that a DPD particle occupies a volume of $90 \AA^{3}$. For the temperature scale, the conversion is less straightforward. The coarse-graining procedure of Groot can be used at any temperature, that is, if we are interested at higher temperature we can again match the compressibility and FloryHuggins solubility parameters at the temperature of interest. This leads to temperature depend parameters which make the

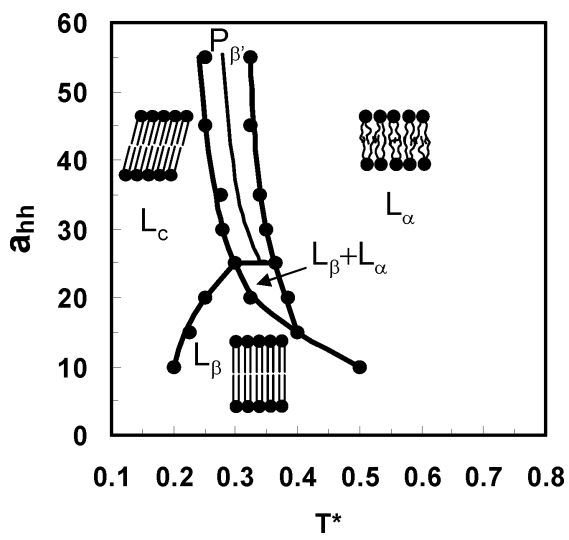

Figure 3. Phase diagram of $h_{3}\left(t_{4}\right)_{2}$ as a function of reduced temperature $T^{*}$ and head-head repulsion parameter $a_{\mathrm{hh}}$. In the narrow region between the $L_{\mathrm{c}}$ phase and the $L_{\alpha}$ phase we find the rippled phase. The thin line corresponds with the condition of $50 \%-50 \%$ of both phases.

interpretation of the results more difficult. To avoid these difficulties, we have used the experimental phase transition temperatures to relate the DPD energy and temperature scales to the experimental system. With these parameters our simulations predict an area per surfactant of $69 \AA^{2}$ of the membrane in the $L_{\alpha}$ or fluid phase, independent of the chain length. This is in good agreement with the experimental values which are in the range 58-72 $\AA^{2}$. For the area per lipid in the gel phase we find $A_{1}=46.6 \AA^{2}$, while the experimental value is $A_{1}=$ $47.2 \AA^{2} .40$

\section{Lipid $h_{3}\left(t_{4}\right)_{2}$}

In this section, we discuss the influence of temperature, tail length, and headgroup repulsion on the phase diagram of doubletail lipids. To facilitate the presentation of our results, we first summarize the computed phase diagram of the lipid $h_{3}\left(t_{4}\right)_{2}$, which consists of three hydrophilic headgroups and two hydrophobic tails with a length of 4 beads. In the next section, we investigate the changes in the phase diagram as a function of the tail length.

We study the phase behavior as a function of temperature and head-head repulsion parameter $a_{\mathrm{hh}}$. We vary the headhead repulsion parameter from $a_{\mathrm{hh}}=10$ to $a_{\mathrm{hh}}=55$. For each value of $a_{\mathrm{hh}}$ we study the temperature behavior of the bilayer by cooling the system in steps of $\Delta T=0.05$. The resulting phase diagram of the lipid $h_{3}\left(t_{4}\right)_{2}$ is given in Figure 3. In these simulations, we observe the low temperature $L_{\mathrm{c}}$ phase and the high temperature $L_{\alpha}$ phase. For low values of $a_{\mathrm{hh}}$ we find that the transition from the $L_{\mathrm{c}}$ phase to the $L_{\alpha}$ phase takes place via the $L_{\beta}$ phase, in which the tails are ordered, but are not tilted with respect to the bilayer normal. At high head-head repulsion $\left(a_{\mathrm{hh}}>25\right)$ the transition $L_{\mathrm{c}} \rightarrow L_{\alpha}$ occurs via the rippled phase $P_{\beta^{\prime}}$.

A. Phase Behavior as a Function of Temperature and Headgroup Interaction. Figure 4 shows the area per lipid, the thickness of the hydrophobic core and the tail order parameter as a function of temperature for various head-head repulsion parameters. At the low-temperature extreme $\left(T^{*}<0.2\right)$, the area per lipid is small and the hydrophobic thickness is large. This indicates that the lipids are tightly packed, which is reflected in the order parameter. The high value of $S_{\text {tail }}$ at low temperatures indicates that the tails are ordered. This tail order parameter does not reach the value of 1 (ordering parallel to the bilayer normal), due to an average tilt angle with respect to the bilayer normal of about $\theta=25^{\circ}$, except for $a_{\mathrm{hh}}=10$ where $\theta=15^{\circ}$. At $T^{*}>0.6$ a gradual increase of $A_{1}$ and decrease of 


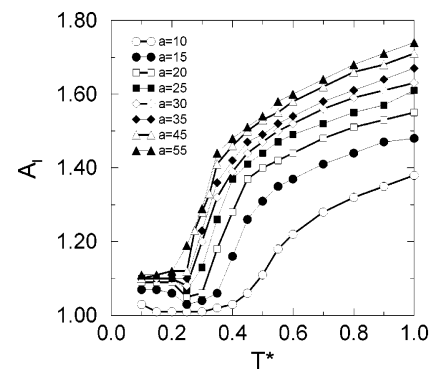

(a)

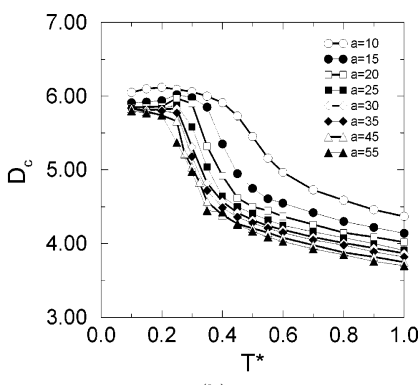

(b)

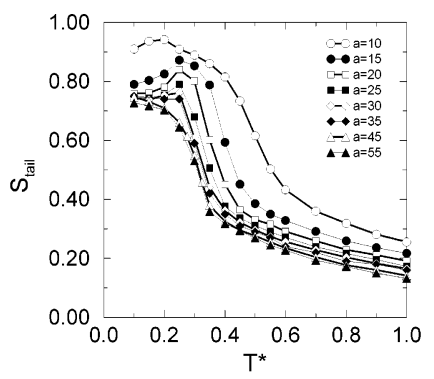

(c)

Figure 4. (a) Area per lipid $A_{\mathrm{l}}$,(b) hydrophobic thickness $D_{\mathrm{c}}$, and (c) tail order parameter $S_{\text {tail }}$ as a function of temperature $T^{*}$ at and headhead repulsion parameter $a_{\mathrm{hh}}$.

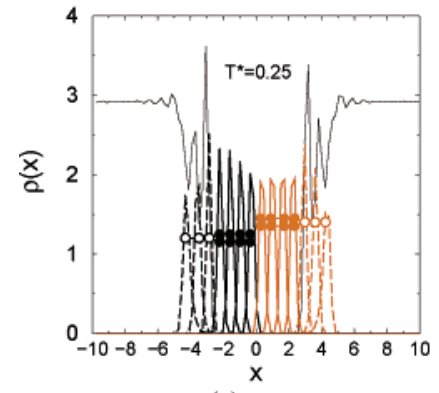

(a)

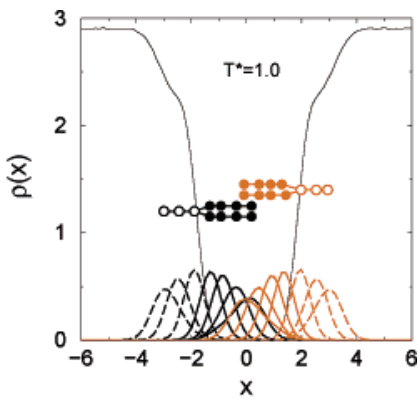

(b)
Figure 5. Density profiles $\rho(x)$ along the bilayer normal $x$ for temperatures (a) $T^{*}=0.25$ and (b) $T^{*}=1.0$. Each line is the density profile for a different bead: full lines are the densities of the tail beads, dashed lines correspond to the head beads and the thin solid line is the density of water, while the colors black and gray represent the lipids of the two monolayers. The dots correspond with the $x$ position of the maximum density, illustrating the positions of the beads in the bilayer.

$D_{\text {c }}$ is observed. At these high temperatures the order of the tail is lost. For the intermediate temperatures we observe a more complex temperature dependence.

In Figure 5, we plotted the density profiles in the direction of the bilayer normal for both temperatures, taking $a_{\mathrm{hh}}=35$ as an example. At high temperature $\left(T^{*}=1.0\right)$ the different tail segments have a low order, reflected in the broad distribution peaks and overlap of the two monolayers, due to disorder of the tails, is observed. This phase corresponds with the fluid $L_{\alpha}$ phase. At a temperature of $T^{*}=0.25$ the peaks are narrow, indicating a high order, and the two monolayers are completely separated. At this temperature the bilayer is in the $L_{\mathrm{c}}$ phase. Due to the high organization of the tails, the headgroups are quite ordered as well and thus the density of water is locally increased. The bulk water (depicted by the thin line) is in the fluid phase. Lowering the temperature even further to $T^{*}=$ 0.1 results in complete freezing of the tails and at this temperature also the water starts to freeze.

The main trends in the curves of the area per lipid, the hydrophobic thickness, and the order parameter can now be explained. At low temperatures, the packing of the tails is the dominating effect: the order in the tails is high and the tails stretch out, which results in a large value for the thickness of the hydrophobic core and a small area per lipid. To minimize contributions to the total energy of both headgroup and tailinteractions, the tails are tilted with respect to the bilayer normal. With increasing temperature the tails loose their order and the collective tilt, and the bilayer becomes fluid. Due to this increasing disorder the area per lipid increases and the hydrophobic thickness decreases.

Till now, we concentrated on the two extreme temperature regions, but a distinction can be made between the phase transition at low head-head repulsion $\left(a_{\mathrm{hh}} \leq 25\right)$ and high head-head repulsion parameters $\left(a_{\mathrm{hh}}>25\right)$. For $a_{\mathrm{hh}}<15$ the system gains energy if a water particle, which is hydrating a head particle, is replaced by another head particle, while for higher repulsion parameters the system gains energy by surrounding the headgroups with water.

For every value of $a_{\mathrm{hh}}$ the tails are in the $L_{\mathrm{c}}$ phase at the lowest temperatures. At low $a_{\mathrm{hh}}$, for which the water particles are expelled from the headgroup region, we observe that at $T^{*}$ $>0.2$ the area per lipid decreases slightly and the thickness increases. At the same time, the order parameter increases. Investigating the tilt angles in these systems shows that at the lowest temperature a collective tilt is present, but with slightly increasing temperature the tilt angle disappears. The disappearance of the tilt angle explains the increase in the order parameters, since this order parameter is calculated with respect to the bilayer normal. In this temperature region, the tails are still ordered and due to the strong head-head interactions the system will form the flat gel phase $L_{\beta}$. For $a_{\mathrm{hh}}>25$ we do not observe the formation of the $L_{\beta}$ phase. At these values of the head-head repulsion the most favorable configuration of the heads is to be surrounded by water and as a result the tails will optimally pack in a tilted configuration.

We investigated the temperature region in which the phase transition occurs more accurately by computing a thickness profile of the bilayer. In Figure 6 we plotted the distribution of the thickness as function of temperature for three different repulsion parameters. We observe that the transition to the $L_{\alpha}$ phase occurs via a narrow region, except for the lowest headhead repulsion parameters of $a_{\mathrm{hh}} \leq 15$, where the transition takes place at once.

For values of $a_{\mathrm{hh}}>25$ the distribution near the transition temperature shows a double peak. The low value corresponds with the thickness of the $L_{\alpha}$ phase and the high value with the thickness of the $L_{\mathrm{c}}$ phase, indicating that there is a coexistence between the two phases. If we simulate exactly at the point where the bilayer consists of $50 \% L_{\mathrm{c}}$ and $50 \% L_{\alpha}$, then we find the contour plot depicted in Figure 7(a). This contour plot shows the thickness of the bilayer as a function of the position in the $y z$ plane. The thick and thin parts of the bilayer alternate, leading to a striped structure. This structure closely resembles the rippled phase $P_{\beta^{\prime}}$ as can also be seen in Figure $7 \mathrm{~b}$. We further investigated this structure and we found that in the thick part the two monolayers are separated and that in the thin arm the end segments of the tails overlap. Computing the tail order parameters of the lipids in the thick and in the thin part, gives that the tails in the thick part of the bilayer are more ordered than the tails in the thin part of the bilayer. Furthermore, the tails in the thick part of the ripple have a tilt angle with respect to the bilayer normal, whereas in the thin part this tilt angle has disappeared. The average orientation of this tilt is parallel to the direction of the ripple. All of these results point out that 


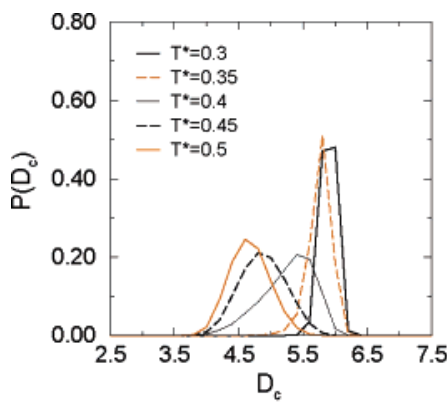

(a) $a_{h h}=15$

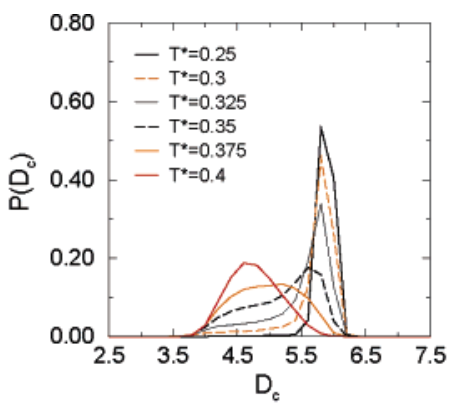

(b) $a_{h h}=20$

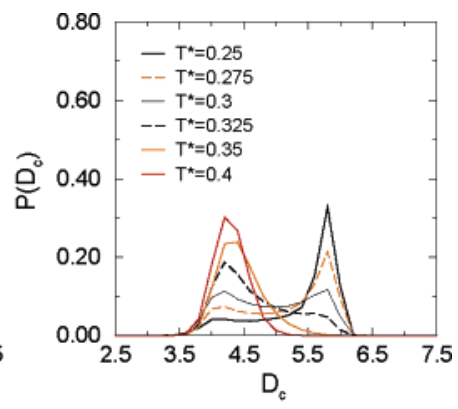

(c) $a_{h h}=45$

Figure 6. Distribution of thicknesses in the bilayer at different temperatures near the transition to the $L_{\alpha}$ phase for three repulsion parameters: (a) $a_{\mathrm{hh}}=15$, (b) $a_{\mathrm{hh}}=20$, and (c) $a_{\mathrm{hh}}=45$.

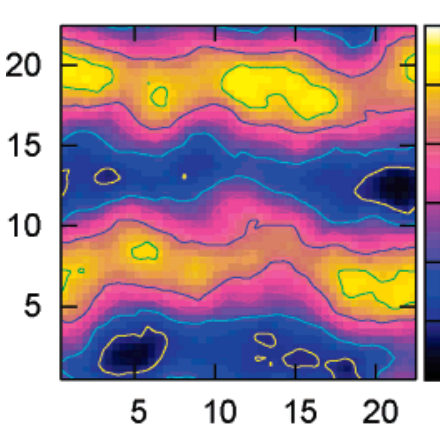

(a)

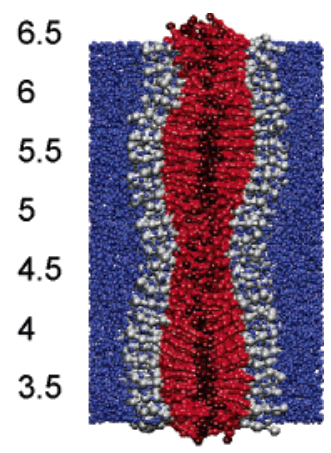

(b)

Figure 7. Structure of the rippled phase with $a_{\mathrm{hh}}=45$ and $T^{*}=0.3$. At this temperature we obtain exactly equal amounts of the $L_{\mathrm{c}}$ and the $L_{\alpha}$ phase. The contourplot (a) shows the thickness of the bilayer as a function of the position in the $y z$, plane where the colors indicate the hydrophobic thickness. (b) is a side view of the bilayer, in which the headgroups are colored black, the tails are gray. The darker color gray is used to indicate the end segments of the tails. The water particles are depicted by smaller spheres.

the rippled phase is a coexistence between the ordered $L_{\mathrm{c}}$ phase and the disordered $L_{\alpha}$ phase.

For $a_{\mathrm{hh}}=20-25$ we do not observe the double peak in the distribution of the thickness near the transition to the fluid $L_{\alpha}$ phase. With increasing temperature the distribution of the thicknesses shifts from the thickness corresponding to the $L_{\beta}$ phase to the thickness observed in the $L_{\alpha}$ phase and all thickness between these two extremes. In Figure 8 the corresponding contourplots are given. At $T^{*}=0.325$ we observe the formation of domains of the $L_{\alpha}$ phase comparable to the domain formation at higher repulsion parameters. With increasing temperature we observe the melting of the bilayer into the $L_{\alpha}$ phase, but this transition is very gradual, as can be seen from Figures 8b,c. We did not find any indication of the formation of a rippled phase.

With these results we are now able to create the phase diagram of the lipid $h_{3}\left(t_{4}\right)_{2}$ (see Figure 3 ). Depending on the headgroup interactions the transition from the low-temperature phase $L_{\mathrm{c}}$ to the fluid phase $L_{\alpha}$ occurs via two different routes. The stability of the $L_{\mathrm{c}}$ phase is determined by the tails, which pack optimally in a tilted configuration. However, the configuration of the headgroups can be far from optimal, depending on the head-head interactions. At low values of $a_{\mathrm{hh}}$ water is expelled from the headgroup region and due to the strong interactions between headgroups the $L_{\beta}$ phase is formed, in which the area per lipid is small, the tails are ordered but no tilt is present. The transition from this phase to the $L_{\alpha}$ phase occurs gradually. For high values of $a_{\mathrm{hh}}$, it is favorable to surround the headgroups with water and the tilt will not disappear. Instead the transition $L_{\mathrm{c}} \rightarrow L_{\alpha}$ will occur via the rippled phase $P_{\beta^{\prime}}$. This rippled structure becomes more stable with increasing hydration of the headgroup.

B. Rippled Phase. To study the formation of the ripple in more detail, we computed the contourplots of the bilayer at different temperatures near the temperature where we find the ripple ( i.e., at the point where we have $50 \%$ of the $L_{\mathrm{c}}$ phase and $50 \%$ of the $L_{\alpha}$ phase) (see Figure 9). At the temperature of $T^{*}=0.275$ the bilayer is mainly in the $L_{\mathrm{c}}$ phase, but domains of the $L_{\alpha}$ phase are formed. At a temperature of $T^{*}=0.325$ we observe that the bilayer is in the $L_{\alpha}$ phase, containing domains of the $L_{\mathrm{c}}$ phase. At both temperatures, these domains are stable and do not fuse into one large domain and the typical rippled structure is not formed.

The rippled structure is more stable with increasing headhead repulsion parameter. At $a_{\mathrm{hh}}=35$ we find the rippled structure only at the temperature where we have $50 \%$ of the $L_{\mathrm{c}}$ phase and $50 \%$ of the $L_{\alpha}$ phase. However, at a repulsion parameter of $a_{\mathrm{hh}}=55$ we find that this structure is stable at a temperature range of $\Delta T^{*}=0.05$ around this temperature. A second effect of increasing the head-head repulsion parameter is on the distance between two ripples. At higher repulsion

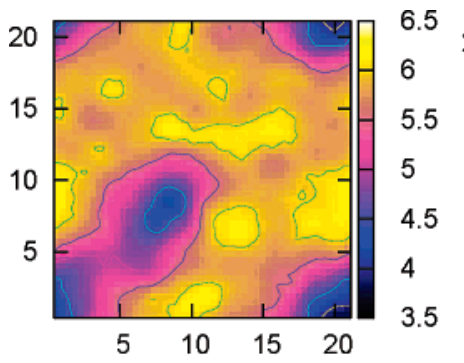

(a) $a_{h h}=20, T^{*}=0.325$

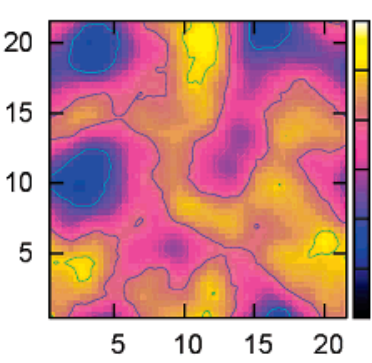

(b) $a_{h h}=20, T^{*}=0.35$

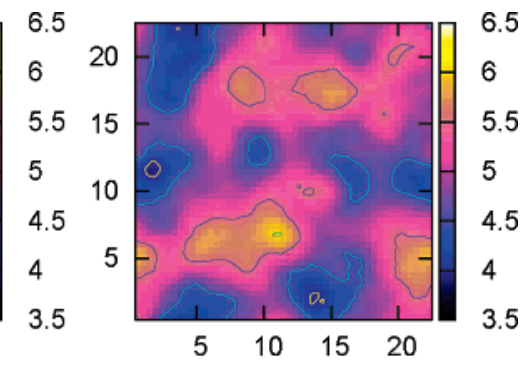

(c) $a_{h h}=20, T^{*}=0.375$

Figure 8. Contourplots of the bilayer at the head-head repulsion $a_{\mathrm{hh}}=20$ at temperatures of (a) $T^{*}=0.325$, (b) $T^{*}=0.35$, and (c) $T^{*}=0.375$. The colors indicate the hydrophobic thickness of the bilayer. 


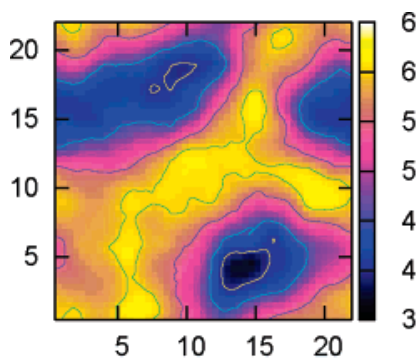

(a) $a_{h h}=45, T^{*}=0.275$

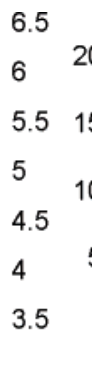$$
275
$$

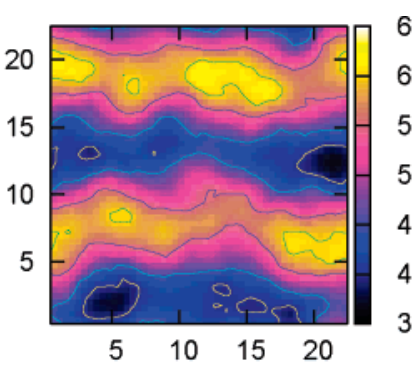

(b) $a_{h h}=45, T^{*}=0.3$

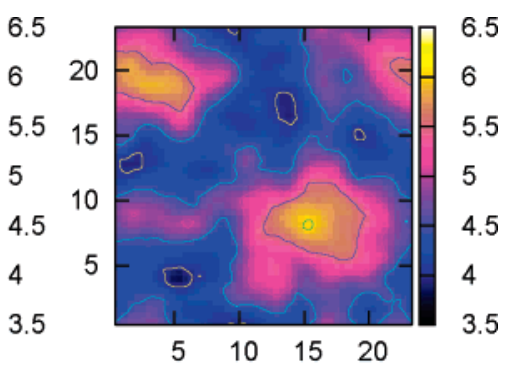

(c) $a_{h h}=45, T^{*}=0.325$

Figure 9. Contourplots of the bilayer at the head-head repulsion $a_{\mathrm{hh}}=45$ at temperatures of (a) $T^{*}=0.275$, (b) $T^{*}=0.3$, and (c) $T^{*}=0.325$. The colors indicate the hydrophobic thickness of the bilayer.

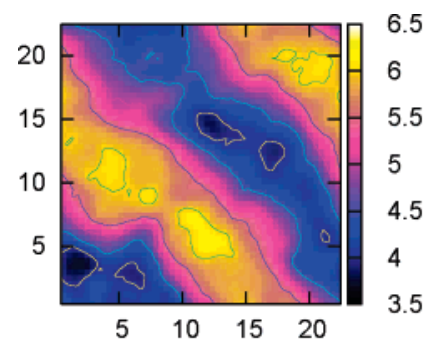

(a)

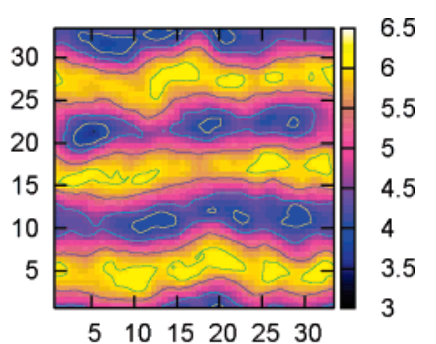

(b)
Figure 10. Contourplots at the head-head repulsion $a_{\mathrm{hh}}=55$ at temperature $T^{*}=0.275$ of (a) a bilayer consisting of 800 lipids and (b) a bilayer consisting of 1800 lipids.

parameters the headgroup is more hydrated, which leads to slightly higher value of the tilt angle in the $L_{\beta^{\prime}}$ phase. As a result, the period of the rippled phase increases slightly with increasing repulsion parameter.

We performed several simulations to test whether the rippled structure that we observe is really a stable phase, or that this structure is induced by the way the simulations are performed. In all simulations, we cooled the system from $T^{*}=1.0$ to $T^{*}$ $=0.1$ in steps of $\Delta T=0.05$. If the rippled phase is the stable phase, it should also be formed if we heat the system up. For $a_{\mathrm{hh}}=45$, at which the rippled phase is observed at $T^{*}=0.3$ (see Figure 9b), we decreased the temperature from $T^{*}=0.325$ to $T^{*}=0.3$ and increased the temperature from $T^{*}=0.275$ to $T^{*}=0.3$. In both cases the rippled structure is observed at $T^{*}$ $=0.3$, while at the lower and the higher temperature no ripple was observed.

We also performed some simulations to test the influence of the system size. For $a_{\mathrm{hh}}=45$, we find the rippled structure in a system containing 800 lipids (Figure 9b). In this system, two "ripples" are observed. Increasing the system size to 1800 lipids, should give the formation of three ripples. However, we find that only two ripples are formed. If we double this system to 6400 lipids, we indeed observe the formation of four ripples.

For $a_{\mathrm{hh}}=55$, we observed that the ripple was formed diagonally in the $y z$-plane of the simulation box (Figure 10(a)). Since we apply periodic boundary conditions in all three directions, one might wonder if this is a rippled phase or just the formation of two domains. Increasing the system size to 1800 lipids shows that in this case the striped structure is again formed parallel to the $y$-axis of the system (Figure 10b), indicating that the rippled phase is the stable phase. Also, in this case, multiplying the system by two leads to a doubling of the number of ripples.

These results show that in the system of 800 lipids, there can be some friction in the distance between the ripples. Increasing the system size leads to the formation of a rippled phase, in which the distance between the ripples is optimal. Once optimized this period of the ripple, there is a linear relation between the system size and the number of ripples. The period of the ripple is very characteristic and depends on the tilt angle of the hydrocarbon tails.

In summary, we observe that the transition from the ordered gel phase to the disordered fluid phase occurs via a coexistence region. For low values of the head-head repulsion parameter $\left(a_{\mathrm{hh}} \leq 25\right)$, there is a coexistence region of the $L_{\beta}$ and the $L_{\alpha}$ phase, in which domains are formed. These domains grow with increasing temperature until the bilayer is completely fluid. Contrary to this behavior, we do not find domain formation for the high values of $a_{\mathrm{hh}}\left(a_{\mathrm{hh}}>25\right)$. The coexistence between the $L_{\beta^{\prime}}$ phase and the $L_{\alpha}$ phase leads to the rippled phase $P_{\beta^{\prime}}$. The period of this ripple depends on the tilt angle of the hydrophobic tails.

\section{Influence of Tail Length}

Now that we have determined the phase diagram of the shortest lipid, it is interesting to investigate how this diagram changes with increasing tail length. We performed simulations on model lipids with tail lengths increasing up to 7 beads in the tail. The resulting phase diagrams are presented in Figure 11. We included the phase diagram of $h_{3}\left(t_{4}\right)_{2}$ for comparison.

In all phase diagrams, we find the phases observed for the shortest model $h_{3}\left(t_{4}\right)_{2}$. However, with increasing tail length we observe the appearance of a third phase, the $L_{\beta^{\prime}}$ phase. This $L_{\beta^{\prime}}$ phase is characterized by less order in the tails than in the $L_{\mathrm{c}}$ phase, a collective tilt with respect to the bilayer normal and no overlap of the two sheets of the bilayer. The temperatureand $a_{\mathrm{hh}}$ stability depends on tail length: the $L_{\beta^{\prime}}$ is more stable if the tails are longer. The appearance of the $L_{\beta^{\prime}}$ phase is in agreement with experimental data of the phase behavior. For DLPC (DiLauroylPC, 12 carbons per tail) the $L_{\beta^{\prime}}$ phase is not observed, while this phase becomes more stable if the tail length is increased from 14 to 20 carbons per tail. ${ }^{1,2}$

With increasing tail length the temperature at which the main transition occurs increases, in agreement with experimental observations. This shift in melting temperature is most significant for the $L_{\beta^{\prime}} \rightarrow L_{\alpha}$ transition, the $L_{\beta^{\prime}} \rightarrow P_{\beta^{\prime}}$ transition, and from the transition of the $L_{\beta}-L_{\alpha}$ coexistence region to the pure $L_{\alpha}$ phase. The transition from the $L_{\mathrm{c}}$ phase to the $L_{\beta^{\prime}}$ is at an almost constant temperature independent of tail length. Thus the increased stability of this phase with increasing tail length is caused by the higher $L_{\beta^{\prime}} \rightarrow P_{\beta^{\prime}}$ transition temperature.

The temperature region, in which the $P_{\beta^{\prime}}$ phase is the stable phase, does not depend on tail length. However, we find an increase of the period of the ripple with increasing tail length. Using a system containing 800 lipids, we observed that with increasing tail length the rippled structure was not formed parallel to the $y$ - or $z$-axis of the system, but diagonally, as was 


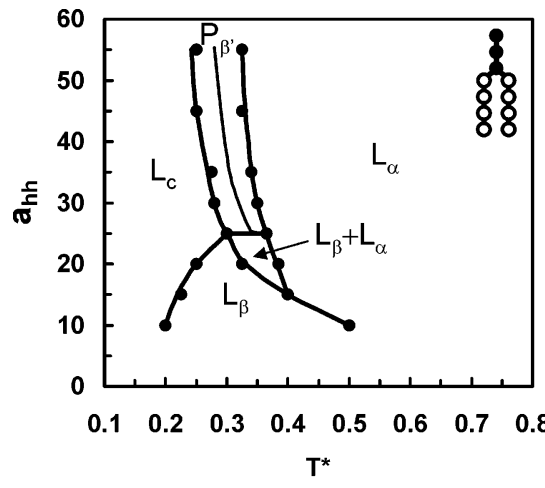

(a)

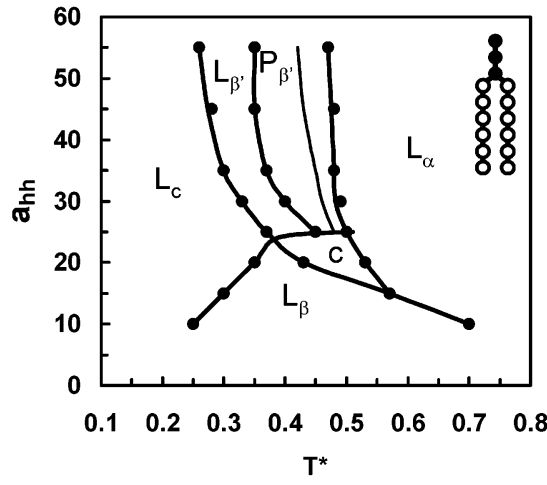

(c)

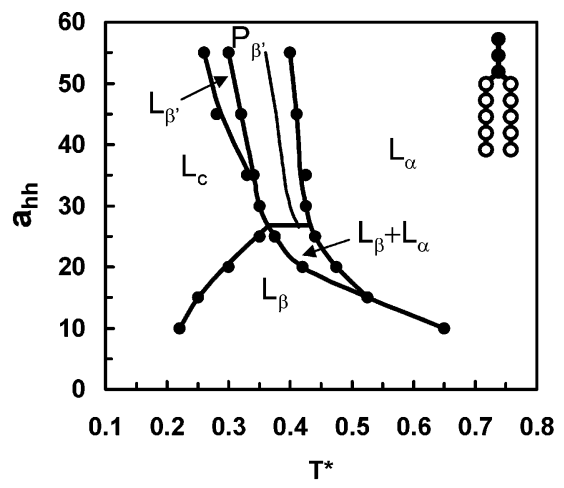

(b)

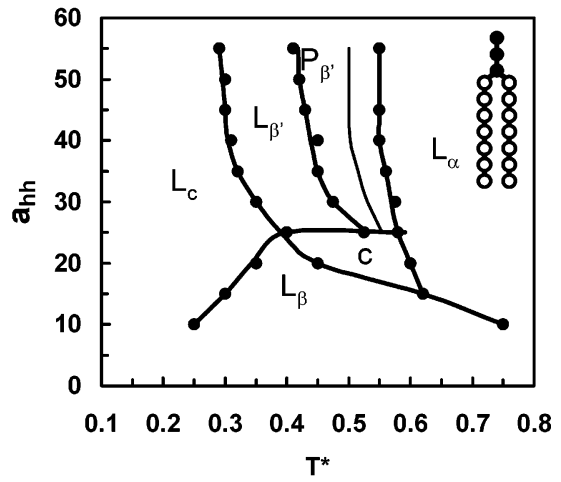

(d)

Figure 11. Phase diagrams of model lipids as a function of head-head repulsion and reduced temperature: (a) $h_{3}\left(t_{4}\right)_{2},\left(\right.$ b) $h_{3}\left(t_{5}\right)_{2},\left(\right.$ c) $h_{3}\left(t_{6}\right)_{2},(d)$ $h_{3}\left(t_{7}\right)_{2}$. In (c) and (d) $\mathbf{c}$ denotes a coexistence region, of which the exact structure was difficult to determine. A description of this phase can be found in the text.

also observed with the lipid $h_{3}\left(t_{8}\right)_{2}$ at $a_{\mathrm{hh}}=55$. By adapting the system size to a maximum of 3200 lipids, the rippled phase was formed for all tail lengths with an increasing period going from 4 to 8 beads in the tail.

For the two longest models studied, it is difficult to determine the structure of the bilayer in the coexistence region at the low head-head repulsion parameters $\left(a_{\mathrm{hh}}<25\right)$. This coexistence region (denoted with $c$ in the phase diagrams of Figure 11c,d) seems to be a coexistence between the $L_{\beta}$, the $L_{\beta^{\prime}}$ phase and the $L_{\alpha}$ phase. With increasing temperatures the average tilt angle first decreases, indicating the $L_{\mathrm{c}} \rightarrow L_{\beta}$ transition. At the second transition the tilt angle again increases till the transition to the $L_{\alpha}$ phase occurs, where no tilt angle is observed. The rippled structure was never observed at these low repulsion parameters.

\section{Discussion}

In this section, we compare the results from our simulations with the experimental data. First, we will discuss the phase diagram as a function of temperature and tail length of the lipids. Then we pay attention to the anomalous swelling, which is observed near the main transition $P_{\beta^{\prime}} \rightarrow L_{\alpha}$, and, finally, we discuss the structure of the rippled phase.

\section{A. Phase Behavior as a Function of Temperature and Tail} Length. Phase behavior as a function of temperature and tail length

In Figure 12 we plotted the transition temperatures as a function of tail length for the typical head-head interaction used by Groot, ${ }^{41} a_{\mathrm{hh}}=35$. The low temperature phase is the highly ordered $L_{\mathrm{c}}$ phase and at high temperatures the $L_{\alpha}$ phase is the stable phase. With increasing temperatures the transition $L_{\mathrm{c}} \rightarrow$ $L_{\alpha}$ goes through different phases, dependent on tail length. For the shortest tail length $(N=4)$ no $L_{\beta^{\prime}}$ phase is observed and

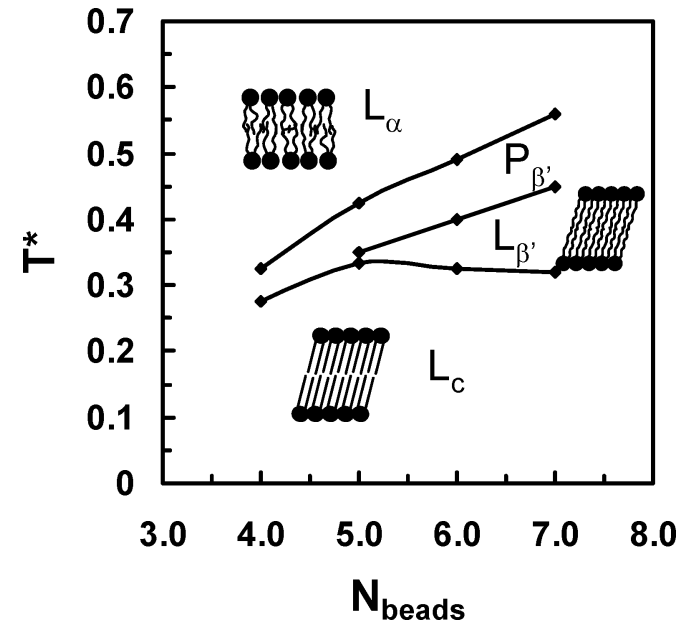

Figure 12. Transition temperatures as a function of tail length for the head-head repulsion parameter $a_{\mathrm{hh}}=35$.

the transition holds $L_{\mathrm{c}} \rightarrow P_{\beta^{\prime}} \rightarrow L_{\alpha}$. For longer tails the $L_{\beta^{\prime}}$ phase is observed between the $L_{\mathrm{c}}$ and $P_{\beta^{\prime}}$ phase. Qualitatively, this phase diagram nicely resembles the experimental phase diagrams. ${ }^{42,43}$ The temperatures of the $L_{\beta^{\prime}} \rightarrow P_{\beta^{\prime}}$ and the $P_{\beta^{\prime}} \rightarrow L_{\alpha^{\prime}}$ transition increase with increasing tail length. The $L_{\beta^{\prime}}$ phase is not observed for the shortest lipid, but for longer tail lengths the stability of this phase increases with increasing tail length. The only difference is that we find a constant temperature region in which the rippled phase is stable, independent of tail length, while experimentally this region decreases and finally disappears with increasing tail length.

We can translate the reduced temperatures to the real temperatures, by, for example, taking the temperatures of the pretransition $L_{\beta^{\prime}} \rightarrow P_{\beta^{\prime}}$ and the main transition $P_{\beta^{\prime}} \rightarrow L_{\alpha^{\prime}}$ of the 
phospholipid DMPC as reference points. DMPC corresponds with the model lipid $h_{3}\left(t_{5}\right)_{2}$. If we take $T^{*}=0.35$ and $T_{\exp }=$ $15.3{ }^{\circ} \mathrm{C}$ for the pretransition temperature and $T^{*}=0.425$ and $T_{\exp }=24.0^{\circ} \mathrm{C}$ for the main transition, we obtain the linear relation $T_{\exp }=116 \times T^{*}-25.3$. This relation leads to large discrepancies from the experimental values for the pre- and main transitions of the other lipids. As an example, we take a model lipid with a tail length of 7 beads. This mesoscopic model corresponds with the phospholipid DSPC, containing 18 carbons in the hydrocarbon chains. From our simulations, we find $T^{*}$ $=0.56$ for the main transition $P_{\beta^{\prime}} \rightarrow L_{\alpha^{\prime}}$, which corresponds with $T_{\exp }=40^{\circ} \mathrm{C}$. However, the main transition of DSPC is experimentally determined at $T_{\exp }=55^{\circ} \mathrm{C} .4^{44}$

At this point, it is important to recall that the parameters have been tuned to reproduce the compressibility of water and the Flory-Huggins solubilities at ambient conditions. The DPD model is too simple to expect that, once these parameters have been fitted at a given temperature, one would, for example, reproduce the compressibility of water at other temperatures. This gives, however, a temperature dependent $a$ parameter which would make the interpretation of our results more complex. Therefore, we do not expect a quantitative agreement.

B. Anomalous Swelling. One of the main questions in the phase behavior of PCs is the observed anomalous swelling (non linear increase of the lamellar repeat distance with temperature) near a phase transition. For long times, this swelling was considered to be a key factor in the formation of the rippled phase. However, it is not clear what causes this anomalous swelling. ${ }^{2,24}$

Experimental work and theories suggest that the swelling could be caused by increased interactions between bilayers due to changes in the Helfrich undulation forces. ${ }^{45-50}$ Approaching the transition temperature $T_{\mathrm{m}}$, the bilayer has a reduced bending rigidity and as a result the fluctuations of the bilayer increase. Due to these increased bilayer fluctuations the steric repulsion between bilayers increases. As a result the thickness of the water layer between two bilayers becomes larger, which causes the anomalous swelling. ${ }^{51,49}$ Another explanation is that the anomalous swelling is mainly caused by an increase of the thickness of the hydrocarbon region. ${ }^{51,24}$ Near the transition temperature, the hydrocarbon chains show a critically straightening. In most papers, a coupling is made between the various explanations: due to a straightening of the tails, the fluctuations of the bilayer increase, which finally results in the anomalous swelling.

In a recent paper, Mason et al. ${ }^{25}$ showed that the anomalous swelling is not coupled to the formation of the rippled phase $P_{\beta^{\prime}}$. By successively adding methyl groups to DiMyristoylPhosphatidylEthanolamine (DMPE, no methyl groups attached to the terminal nitrogen) to form DMPC (three methyl groups attached) it is observed that anomalous swelling occurs in the case of mmDMPE (monomethyl-DMPE) and dmDMPE (dimethyl-DMPE), while bilayers of these phospholipids undergo a transition into the flat gel phase $\left(L_{\beta}\right)$ rather than into the rippled phase.

In our simulations, we calculated the contribution of the hydrophobic thickness $\left(D_{\mathrm{c}}\right)$ and the thickness of the headgroup region $\left(D_{\mathrm{h}}\right)$ to the swelling for $h_{3}\left(t_{5}\right)_{2}$ for different repulsion parameters. Figure 13(a) shows the hydrophobic thickness and the bilayer thickness $D_{\mathrm{b}}$ as a function of temperature and in Figure $13 \mathrm{~b}$ the thickness of the headgroup region is plotted. We observe a large increase of the hydrophobic thickness, which may be responsible for the swelling of the bilayer.

The behavior of the hydrophobic thickness is closely related to the phase behavior of the bilayer (see Figure 11b). For the
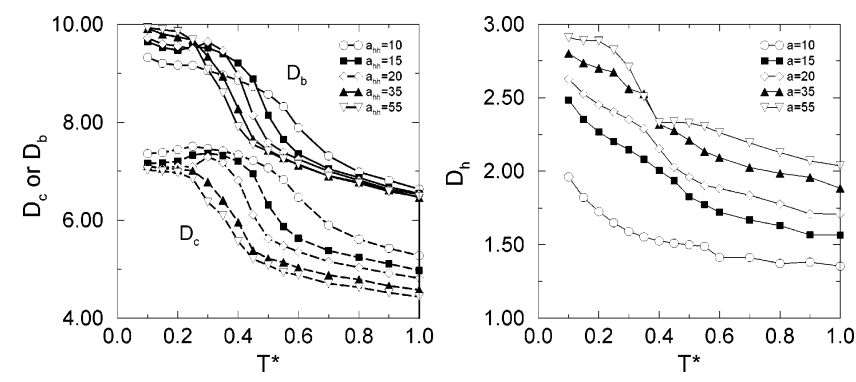

Figure 13. (a) Bilayer thickness $\left(D_{\mathrm{b}}\right)$ (solid lines), thickness of the hydrophobic region $\left(D_{\mathrm{c}}\right)$ (dashed lines), and (b) thickness of the headgroup region $\left(D_{\mathrm{h}}\right)$ as a function of temperature for various headhead repulsion parameters of the $h_{3}\left(t_{5}\right)_{2}$ lipids.

lowest repulsion parameter $\left(a_{\mathrm{hh}}=10\right)$, we observe upon decreasing temperature the transitions $L_{\alpha} \rightarrow L_{\beta} \rightarrow L_{\mathrm{c}}$. Decreasing the temperature in the $L_{\alpha}$ phase results in an increased ordering of the chains and in the $L_{\beta}$ phase the chains are ordered and do not show a tilt. Further decrease of the temperature gives the $L_{\mathrm{c}}$ phase and because of the tilt, the hydrophobic thickness is smaller, which explains the maximum in the curve. This maximum is not present in the curve for the bilayer thickness $D_{\mathrm{b}}$. Figure $13 \mathrm{~b}$ shows that for $a_{\mathrm{hh}}=10$ decreasing the temperature straightens the heads and, in particular, in the $L_{\mathrm{c}}$ phase this increase in the thickness of the head region completely compensates the decrease of the hydrophobic thickness.

For higher values of $a_{\mathrm{hh}}$, but still for $a_{\mathrm{hh}} \leq 25$, the main trend is equal to the trend observed for $a_{\mathrm{hh}}=10$. Figure $11 \mathrm{~b}$ shows that the temperature range, for which the $L_{\beta}$ phase is stable, decreases. Hence, the increase of the hydrophobic thickness in the $L_{\beta}$ phase occurs in a much narrower temperature interval and therefore gives a sharp increase of $D_{\mathrm{c}}$. For $a_{\mathrm{hh}}=20$, the decrease of $D_{\mathrm{c}}$ in the $L_{\mathrm{c}}$ phase is not completely compensated by the increase of the thickness of the headgroups $\left(D_{\mathrm{h}}\right.$, see Figure 13b) and for this headgroup interaction, we observe a decrease of the bilayer thickness.

For $a_{\mathrm{hh}}>25$, for which the ripples phase $P_{\beta^{\prime}}$ is observed, we find a strong increase of the hydrophobic thickness associated to the $L_{\alpha} \rightarrow P_{\beta^{\prime}}$ transition. We also observe a discontinuous increase in the thickness of the headgroup region, located at the point where the $L_{\alpha} \rightarrow P_{\beta^{\prime}}$ transition occurs. Ordering of the tails and the headgroups occurs simultaneously. Due to the strong hydration of the headgroups at high $a_{\mathrm{hh}}$ the headgroups will stretch into the water phase, such that each segment is surrounded by water. This larger increase in $D_{\mathrm{h}}$ causes the change in order of the curves representing the bilayer thickness in the low-temperature region.

For all values of $a_{\mathrm{hh}}$ the increase of the bilayer thickness is mainly a consequence of the increase of the hydrophobic thickness. For $a_{\mathrm{hh}}>25$ the larger increase of the bilayer thickness is caused by a relatively larger contribution of the thickness of the headgroup region.

We observe for the model lipid $h_{3}\left(t_{5}\right)_{2}$, which corresponds to the phospholipid DMPC, that increasing $a_{\mathrm{hh}}$ from a very low value to a value above the triple point results in similar swelling curves to the curves obtained by increasing the size of the headgroup by adding successively methyl groups to form DMPC from DMPE. ${ }^{25}$ Increasing the number of methyl groups in the headgroups corresponds with increasing the repulsion between the lipid headgroups, since the steric hindrance increases with increasing number of methyl groups and the ionic interactions decrease as the headgroup is larger. For DMPE, which corresponds to a lipid with head-head repulsion parameter $a_{\mathrm{hh}}=$ 10, the hydrophobic thickness increases gradually at the 
transition $L_{\alpha} \rightarrow L_{\beta}$. The swelling curves of mmDMPE and dmDMPE, corresponding with $a_{\mathrm{hh}}=15$ and $a_{\mathrm{hh}}=20$, respectively, show a maximum near the main transition. The maximum in bilayer thickness for dmDMPE is sharper than for mmDMPE. ${ }^{25}$ In our simulations, we show that the occurrence of this maximum is due to an increase of the hydrophobic thickness, caused by the formation of the $L_{\beta}$ phase. Since the stability of this $L_{\beta}$ phase decreases with increasing head-head repulsion, the maximum becomes sharper until the triple point is reached at $a_{\mathrm{hh}}=25$. For the PC lipids, corresponding with the high values of $a_{\mathrm{hh}}\left(a_{\mathrm{hh}}>25\right)$, the experimentally obtained curves show a sharp maximum at the main transition for the shorter chain lengths. For the longer tail lengths, this maximum disappears. ${ }^{48,50}$ We observe a large increase of the bilayer thickness for all lipid tail lengths, caused by a simultaneously increase of the hydrocarbon thickness and the thickness of the headgroup region. We do not observe a maximum in the curve for the shorter lipids.

Only for DMPC, which is above the triple point, is a rippled phase observed, which reinforces the conclusion of Mason et $\mathrm{al}^{25}$ that the anomalous swelling of mmDMPE, and dmDMPE is not related to the rippled phase. Our simulations show that in all cases the (anomalous) swelling is the consequence of changes of the conformation of the hydrocarbon tails. Of course, experiments are often performed on multiple layers and changing the temperature may also change the amount of water between the layers. Also, the electrostatic interactions between the lipid headgroups can play a crucial role in the swelling of the bilayer. ${ }^{47,52}$ These effects are not included in our simulations.

C. Structure of the Rippled Phase. One of the main questions in the phase diagram of a phospholipid bilayer is the existence of the rippled phase, since this corrugated phase lies between the two flat phases $L_{\beta^{\prime}}$ and $L_{\alpha}$. Besides much experimental work, a lot of theoretical and modeling studies are devoted to the nature of the rippled phase. Different approaches are used to model the rippled phase. Macroscopic theories regard the bilayer as a whole and explanations can thus be found in the elasticity and the curvature of the membrane. ${ }^{5,10,12,14,19,28,46}$ Microscopic theories explain the existence of the rippled phase in terms of the packing properties of individual molecules. ${ }^{4,7,13,20}$ In these studies, the formation of the ripple is often attributed to a packing competition between the lipid headgroups and the hydrocarbon chains. Also, a combination of these two approaches is possible: it is proposed that competition exists between macroscopic curvature and microscopic properties of the bilayer. ${ }^{17,23,11} \mathrm{~A}$ third approach is the approach in which interbilayer interactions are taken into account in the formation of the ripple. ${ }^{13}$

The general picture of the rippled phase is that the shape is an asymmetric sawtooth, with a difference in thickness between the long and the short arm. ${ }^{5-9}$ However, there are also studies in which the shape of the ripple is sinusoidal.,10-13 The wavelength of the ripples is in the range from 120 to $160 \AA$ if a bilayer is heated from the $L_{\beta^{\prime}}$ phase. This wavelength increases with increasing length of the hydrocarbon tails..$^{6,7,53,54}$ In this section, we compare the results of our simulations with some of the experimental and theoretical studies on the structure of the rippled phase. We do not pay attention to the sawtooth or sinusoidal shape of the ripple, since much larger systems are needed to observe the typical sawtooth.

In most experimental work, the sample is not a unilamellar system but a multilayer. It is assumed that the ripples occur due to bilayer-bilayer interactions, which are mediated by the lipid headgroups. In an AFM study on supported double DPPC- bilayers on mica Fang and Yang ${ }^{26}$ detected the existence of a ripple structure in the upper bilayer of the double-bilayer regions only. This indicates that the bilayer-bilayer interaction might be responsible for the formation of the ripple structures. However, also in unilamellar systems the pretransition is found. Mason et al. ${ }^{27}$ argue that in the AFM study the undulations are suppressed by the substrate and they provide evidence that the rippled phase exists in large unilamellar vesicles of DPPC. Another study by Takeda et al. ${ }^{28}$ shows that the ripple structure appears in a system if the thickness of the water layer between the lipid bilayers is increased by the addition of salt, indicating that the ripple originates mainly in the intralayer interactions. In these unilamellar systems, the transition is broader and less separated from the main transition. ${ }^{12,55}$

All our simulations are performed on a single bilayer, and we observe the rippled phase in all systems, provided that $a_{\mathrm{hh}}$ $>25$. Although we apply periodic boundary conditions in all three directions, there is no bilayer-bilayer interaction. We impose that the thickness of the water layer between two bilayers is at least $4 r_{\mathrm{c}}$ to guarantee that two periodic images do not have any interactions in the $x$-direction. To test whether the results change if the bilayers do interact, we performed simulations on a multilayer system at $a_{\mathrm{hh}}=35$ in the temperature range $T^{*}$ $=0.1$ to $T^{*}=0.7$. These simulations gave the same results as were obtained for a single bilayer, indicating that interbilayer interactions are not the key factor in the formation of a rippled structure.

It is difficult to determine directly the structure of the two arms of the ripple, which differ in thickness, experimentally. ${ }^{56}$ For example, Sun et al. ${ }^{7}$ assume that the X-ray diffraction patterns are best fitted with a model in which the asymmetry of the bilayer height profile is the dominant feature. The major side of the ripple is similar to the $L_{\beta^{\prime}}$ phase, while the minor side may be more the fluid $L_{\alpha}$ phase. The formation of the ripple due to the coexistence between these two phases is also proposed in many other experimental and theoretical studies. ${ }^{4,19-23}$ However, since it was found that in the $P_{\beta^{\prime}}$ phase the chains are mainly frozen in an all-trans configuration, ${ }^{18}$ the explanation of coexistence is less probable and the difference in the existence of the ripple is attributed to a change in tilt angle and/or elastic properties. ${ }^{12-17}$ Sengupta et al. ${ }^{17}$ conclude that the asymmetry of the ripple is not caused by an asymmetry of the height profile, but that the difference in the bilayer thickness is the primary feature. In this model, the height profile is symmetric and the differences in the thickness are attributed to a mean tilt of the hydrocarbon chains.

Our simulations show that the thickness in the two parts of the ripple is different, due to coexistence of the $L_{\mathrm{c}}$ or $L_{\beta^{\prime}}$ phase and the $L_{\alpha}$ phase. In the thick part $\left(L_{\mathrm{c}}\right.$ or $\left.L_{\beta^{\prime}}\right)$, the tails have a preferred tilt, while in the thin part $\left(L_{\alpha}\right)$ this tilt has disappeared. The contribution of the $L_{\alpha}$ phase in the $P_{\beta^{\prime}}$ phase increases with increasing temperature (see Figures 6 and 9), and at the condition of $50-50 \%$ of both phases $\left(L_{\mathrm{c}}\right.$ or $L_{\beta^{\prime}}$ and $\left.L_{\alpha}\right)$ we find a structure that is similar to the rippled structure. This indicates that both the pretransition and main transition are caused by the same effect of chain melting. ${ }^{4}$ In the case of coexistence, the chains are not frozen in an all-trans conformation as was proposed by Cameron. ${ }^{18}$ However, spectroscopic ${ }^{21}$ and diffusion studies ${ }^{57}$ have shown the existence of a significant fraction of disordered chains, supporting the presence of the $L_{\alpha}$ phase.

A surprising aspect of the rippled phase is that, unlike ordinary coexistence, the system does not minimize the total interfacial area formed by the two phases. Our simulations show that the head-head interaction is a key factor in the formation 


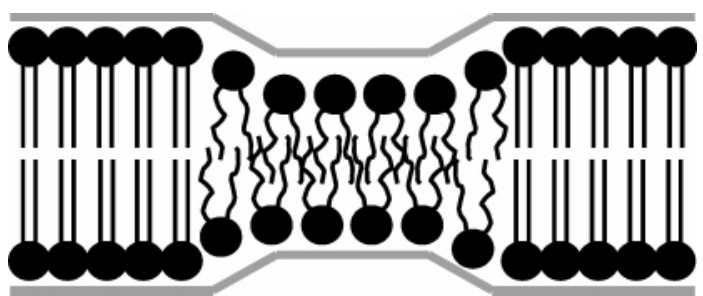

Figure 14. Schematic Figure of the rippled phase. Because of the coexistence between the thick $\left(L_{\mathrm{c}}\right.$ or $L_{\beta^{\prime}}$ ) and thin $\left(L_{\alpha}\right)$ phases, the system can lower its energy by increasing the number of interfaces. In this way the total water headgroup area is increased at the interfacial region.

of the ripple: if $a_{\mathrm{hh}}<25$ we do not observe the rippled phase. For $a_{\mathrm{hh}}>25$, the system can gain energy if more headgroups are exposed to water. In the coexistence region the headgroup water contact area is locally increased (see Figure 14), hence by increasing the number of interfaces the system can lower its energy. We also observe this tendency to increase the headgroup water contact area, if we perform simulations in the region where there is coexistence of the two phases, but not at the condition of 50-50\% $L_{\beta^{\prime}}-L_{\alpha}$. We observe that at different ratios of $L_{\beta^{\prime}}$ $-L_{\alpha}$ more than one domain of one phase in the dominating phase is formed (see Figure 9). In this way, the number of headgroups exposed to water is larger than if only one domain is formed.

Obviously, the system can lower its energy by increasing the number of interfaces in the rippled structure. The total number of interfaces, however, will be limited by the repulsive forces between the ripples. The origin of this force is the elastic energy, which tends to minimize the curvature of the interface between the thick and thin parts. This aspect will depend on the tail length; the longer the tails, the larger the thickness that has to be crossed. This explains why we observe that the period of the ripple increases with tail length. Within the thick part of the ripple, the average orientation of the tilt is parallel to the direction of the ripple, which is an important factor in stabilizing a linear interface.

It is interesting to compare these results with the continuum Landau theory of Lubensky and MacKintosh, ${ }^{12}$ in which a rippled phase occurs due to the coupling of molecular tilt to the membrane curvature if the longitudinal elastic constant is negative. Our results show that the microscopic origin of this negative constant is the surface area of the heads, which is not compatible with the lateral density of the tails. This confirms the reasoning by Carlson and Sethna. ${ }^{13}$ They argue that the packing competition between the headgroups and chains is the essential feature for two reasons. First, the ripple does not occur in bilayers consisting of phospholipids with smaller headgroups and second, the pretransition temperature increases as the hydrocarbon chain length is increased, which suggests that the attraction between the chains is important.

\section{Conclusions}

In this investigation, we studied the phase behavior of phospholipid bilayer, and especially the formation of the rippled phase, as a function of temperature, headgroup interactions, and tail length. The formation of the rippled phase, its relation with anomalous swelling of these membranes, and the structure of the ripple observed about 30 years ago has attracted the attention of many researches. Many different theories have been put forward to explain the formation of this phase, but a molecular explanation is still lacking. Therefore, we perform computer simulation on a mesoscopic model of a lipid, that consists of a headgroup of three hydrophilic beads and two hydrophobic tails varying in length from 4 to 7 beads.

We showed that we can reproduce the experimentally observed phases. At low temperatures the $L_{\mathrm{c}}$ phase is stable, in which the tails are highly ordered and show a tilt with respect to the bilayer normal. Increasing temperature leads to the melting of the bilayer, which goes through different phases, dependent on the headgroup interactions. For low values of the headhead repulsion parameters, the headgroups want to expel water and as a consequence the transition to the fluid $L_{\alpha}$ phase takes place via the flat gel phase $L_{\beta}$, in which the tilt has disappeared. For high values of the head-head repulsion parameter, we find a coexistence region of the gel phase and the fluid phase. We observe the rippled structure $\left(P_{\beta^{\prime}}\right)$ in a narrow region around the line where we have approximately $50 \% L_{\mathrm{c}}$ or $L_{\beta^{\prime}}$ phase and $50 \% L_{\alpha}$ phase. For longer tails, this phase is preceded by the $L_{\beta^{\prime}}$ phase. The stability of this phase increases with increasing tail length.

A key factor in the understanding of the rippled phase, is a frustration induced by the optimal surface area of the heads which is not compatible with the optimal lateral density of the tails. For high values of $a_{\mathrm{hh}}$, the system can gain energy if more headgroups are exposed to water and therefore, at the condition of 50-50\% material of both phases, the space filling problem leads to a striped solution. Taking into account the curvature constrain, the period of the ripple increases with increasing tail length. The anomalous swelling, observed at the $P_{\beta^{\prime}} \rightarrow L_{\alpha}$, is caused by conformational changes of the lipid tails, but is not directly related to the rippled phase.

Acknowledgment. These investigations are supported by The Netherlands Research Council for Chemical Sciences (CW) and by The Netherlands Organization for Scientific Research (NWO) through PIONIER. B.S. would like to thank David Chandler for continuous advice, inspiration, and support.

\section{References and Notes} 145 .

(1) Koynova, R.; Caffrey, M. Biochim. Biophys. Acta 1998, 1376, 91-

(2) Nagle, J. F.; Tristram-Nagle, S. Biochim. Biophys. Acta 2000, 1496, 159-195.

(3) Jørgensen, K. Biochim. Biophys. Acta 1995, 1240, 111-114.

(4) Heimburg, T. Biophys. J. 2000, 78, 1154-1165.

(5) Tardieu, A.; Luzzatti, V.; Reman, F. C. J. Mol. Biol. 1973, 75, 711-733. 976.

(6) Woodward, J. T.; Zasadzinski, J. A. Biophys. J. 1997, 72, 964-

(7) Sun, W.-J.; Tristram-Nagel, S.; Suter, R. M.; Nagle, J. F. Proc. Natl. Acad. Sci. U.S.A. 1996, 93, 7008-7012.

(8) Sengupta, K.; Raghunathan, V. A.; Katsaras, J. Phys. Rev. E 1999, $59,2455-2457$

(9) Zasadzinsky, J. A. N.; Schneir, J.; Gurley, J.; Elings, V.; Hansma, P. K. Science 1988, 239, 1013-1015.

(10) Goldstein, R. E.; Leibler, S. Phys. Rev. Lett. 1988, 61, 2213-2216.

(11) Doniach, S. J. Chem. Phys. 1979, 70, 4587-4596.

(12) Lubensky, T. C.; MacKintosh, F. C. Phys. Rev. Lett. 1993, 71, $1565-1568$

(13) Carlson, J. M.; Sethna, J. P. Phys. Rev. A 1987, 36, 3359-3374.

(14) Chen, C. M.; Lubensky, T. C.; Mackintosh, F. C. Phys. Rev. E 1995, 51, 504-513.

(15) Larsson, K. Chem. Phys. Lipids 1977, 20, 225-228.

(16) Chen, C. M.; Mackintosh, F. C. Phys. Rev. E 1996, 53, 49334943.

(17) Sengupta, K.; Raghunathan, V. A.; Hatwalne, Y. Phys. Rev. Lett. 2001, 87, art. no. 055705 .

(18) Cameron, D. G.; Casal, H. L.; Mantsch, H. H. Biochemistry 1980, $19,3665-3672$.

(19) Marder, H. L. M. andFrisch; Langer, J. S.; McConnell, H. M. Proc. Natl. Acad. Sci. U.S.A. 1984, 81, 6559-6561.

(20) Rappolt, M.; Pabst, G.; Rapp, G.; Kriechbaum, M.; Amenitsch, H.; Krenn, C.; Bernstorff, S.; Laggner, P. Eur. Biophys. J. 2000, 29, 125-133. 
(21) Wittebort, R. J.; Schmidt, C. F.; Griffin, R. G. Biochemistry 1981, 20, 4223-4228.

(22) Banerjee, S. Physica A 2002, 308, 89-100.

(23) Falkovits, M. S.; Seul, M.; Frisch, H. L.; McConnell, H. M. Proc. Natl. Acad. Sci. U.S.A. 1982, 79, 3918-3921.

(24) Nagle, J. F.; Petrache, H. I.; Gouliaev, N.; Tristram-Nagle, S.; Liu, Y.; Suter, R. M.; Gawrisch, K. Phys. Rev. E 1998, 58, 7769-7776.

(25) Mason, P. C.; Nagle, J. F.; Epand, R. M.; Katsaras, J. Phys. Rev. E 2001, 63, art. no. 030902(R).

(26) Fang, Y.; Yang, J. J. Phys. Chem. 1996, 100, 15614-15619.

(27) Mason, P. C.; Gaulin, B. D.; Epand, R. M.; Wignall, G. D.; Lin, J. S. Phys. Rev. E 1999, 59, 3361-3367.

(28) Takeda, T.; Ueno, S.; Kobayashi, H.; Komura, S.; Seto, H.; Toyoshima, Y. Physica B 1995, 213\&214, 763-765.

(29) Kranenburg, M.; Laforge, C.; Smit, B. Phys. Chem. Chem. Phys. 2004, 6, 4531-4534

(30) Groot, R. D.; Warren, P. B. J. Chem. Phys. 1997, 107, 44234435 .

(31) Frenkel, D.; Smit, B. Understanding Molecular Simulations: from Algorithms to Applications, 2nd ed.; Academic Press: San Diego, 2002.

(32) Kranenburg, M.; Nicolas, J. P.; Smit, B. Phys. Chem. Chem. Phys.

2004, 6, 4142-4151.

(33) Kranenburg, M.; Smit, B. FEBS Lett. 2004, 568, 15-18.

(34) Kranenburg, M.; Vlaar, M.; Smit, B. Biophys. J. 2004, 87, 15961605 .

(35) Goetz, R.; Lipowsky, R. J. Chem. Phys. 1998, 108, 7397-7409.

(36) Goetz, R.; Gompper, G.; Lipowsky, R. Phys. Rev. Lett. 1999, 82, $221-224$. 10

(37) Venturoli, M.; Smit, B. Phys. Chem. Commun. 1999, 2, art. no.

(38) Kranenburg, M.; Venturoli, M.; Smit, B. Phys. Rev. E 2003, 67, art. no. 060901

(39) Kranenburg, M.; Venturoli, M.; Smit, B. J. Phys. Chem. B 2003 , 107, 11491-11501.
(40) Tristram-Nagle, S.; Liu, Y.; Legleiter, J.; Nagle, J. F. Biophys. J 2002, 83, 3324-3335.

(41) Groot, R. D. Langmuir 2000, 16, 7493-7502.

(42) Cunningham, B. A.; Brown, A.-D.; Wolfe, D. H.; Williams, W. P.; Brain, A. Phys. Rev. E 1998, 58, 3662-3672. 1890.

(43) Tenchov, B.; Koynova, R.; Rapp, G. Biophys. J. 2001, 80, 1873-

(44) Gennis, R. B., Biomembranes. Molecular Structure and Function; Springer-Verlag: New York, 1989.

(45) Pabst, G.; Katsaras, J.; Raghunathan, V. A.; Rappolt, M. Langmuir 2003, 19, 1716-1722.

(46) Pabst, G.; Katsaras, J.; Raghunathan, V. A. Phys. Rev. Lett. 2002, 12 , art. no. 128101 .

(47) Korreman, S. S.; Posselt, D. Euro. Biophys. J. 2001, 30, 121128

(48) Korreman, S. S.; Posselt, D. Euro. Phys. J. E 2000, 1, 87-91.

(49) Chen, F. Y.; Hung, W. C.; Huang, H. W. Phys. Rev. Lett. 1997, 79, 4026-4029.

(50) Lemmich, J.; Mortensen, K.; Ipsen, J. H.; Honger, T.; Bauer, R.; Mouritsen, O. G. Phys. Rev. E 1996, 53, 5169-5180.

(51) Zhang, R.; Sun, W.; Traistram-Nagle, S.; Headrick, R. L.; Suter,

R. M.; Nagle, J. F. Phys. Rev. Lett. 1995, 74, 2832-2835.

(52) Misbah, C.; Duplat, J.; Houchmandzadeh, B. Phys. Rev. Lett. 1998 , $80,4598-4601$

(53) Janiak, M. J.; Small, D. M.; Singer, M. A. Biochemistry 1976, 15, 4575-4580.

(54) Luna, E. J.; McConnell, H. M. Biochim. Biophys. Acta 1977, 466, $381-392$.

(55) Heimburg, T. Biochim. Biophys. Acta 1998, 1415, 147-162.

(56) Nagle, J. F.; Tristram-Nagle, S. Curr. Opin. Struct. Biol 2000, 10, $474-480$.

(57) Schneider, M. B.; Chan, W. K.; Webb, W. W. Biophys. J. 1983, $43,157-165$ 